\title{
Erratum: Valley magnetism, nematicity, and density wave orders in twisted bilayer graphene [Phys. Rev. B 102, 125120 (2020)]
}

\author{
Dmitry V. Chichinadze, Laura Classen, and Andrey V. Chubukov $\odot$ \\ (Received 17 December 2020; published 4 January 2021)
}

DOI: 10.1103/PhysRevB.103.039901

In the original paper, we erroneously neglected the interaction between fermionic densities in different valleys in the diagrams for intravalley charge orders. Although this interaction does not change the hierarchy of previously reported ordered states, it introduces an additional attraction in the $s^{ \pm}$charge Pomeranchuk channel (a density order with the opposite sign in the two valleys).

The correct result is that in both six-patch and twelve-patch models, the coupling in the $s^{ \pm}$charge Pomeranchuk channel is degenerate with the one in the $s$-wave spin Pomeranchuk channel, responsible for valley magnetism. Our discussion of the Landau free energy for spin Pomeranchuk order is not affected as the system develops either spin or charge (valley) order, but not both.

In practical terms, the coupling matrix $\Lambda_{\mathrm{CPom}, 0}$ in Eq. (21) for the six-patch model should be revised to

$$
\Lambda_{\mathrm{CPom}, 0}=\left(\begin{array}{cccccc}
-u & g-2 u & g-2 u & -2 u & -2 u & -2 u \\
g-2 u & -u & g-2 u & -2 u & -2 u & -2 u \\
g-2 u & g-2 u & -u & -2 u & -2 u & -2 u \\
-2 u & -2 u & -2 u & -u & g-2 u & g-2 u \\
-2 u & -2 u & -2 u & g-2 u & -u & g-2 u \\
-2 u & -2 u & -2 u & g-2 u & g-2 u & -u
\end{array}\right),
$$

and accordingly, the correct $s$-wave eigenvalues are

$$
\lambda_{\mathrm{CPom}}^{s}=\Pi_{++}(0)(2 g-11 u), \quad \lambda_{\mathrm{CPom}}^{s^{ \pm}}=\Pi_{++}(0)(u+2 g) .
$$

Similarly, Eq. (32) should be revised to

$$
\Lambda_{\mathrm{CDW}}\left(\boldsymbol{Q}_{m}\right)=\left(\begin{array}{cccccc}
u-2 g & 0 & 0 & -2 g & 0 & 0 \\
0 & u-2 g & 0 & 0 & -2 g & 0 \\
0 & 0 & u-2 g & 0 & 0 & -2 g \\
-2 g & 0 & 0 & u-2 g & 0 & 0 \\
0 & -2 g & 0 & 0 & u-2 g & 0 \\
0 & 0 & -2 g & 0 & 0 & u-2 g
\end{array}\right),
$$

so that the eigenvalues are now,

$$
\begin{gathered}
\lambda_{\mathrm{CDW}}^{+}\left(\boldsymbol{Q}_{m}\right)=\Pi_{++}\left(\boldsymbol{Q}_{m}\right)(u-4 g), \\
\lambda_{\mathrm{CDW}}^{-}\left(\boldsymbol{Q}_{m}\right)=\Pi_{++}\left(\boldsymbol{Q}_{m}\right) u .
\end{gathered}
$$

They remain subleading, but $\lambda_{\mathrm{CDW}}^{-}\left(\boldsymbol{Q}_{m}\right)$ is now degenerate with $\lambda_{\mathrm{SDW}}\left(\boldsymbol{Q}_{m}\right)$ in Eq. (33).

Intravalley charge orders in the 12-patch model also receive additional corrections, and Eqs. (72) and (75)-(78) must be revised accordingly.

The outcome is the same as in the six-patch model: The hierarchy of leading instabilities remains the same, but the coupling in the $s^{ \pm}$charge Pomeranchuk channel becomes degenerate with the one in the $s$-wave spin Pomeranchuk channel, and the eigenvalues in some CDW and SDW channels become degenerate. 